\title{
Piezoelectricity in Lead-Zirconate-Titanate Ceramics - Extrinsic and Intrinsic Contributions
}

\author{
Johannes Frantti and Yukari Fujioka \\ Aalto University School of Science and Technology \\ Department of Applied Physics \\ Finland
}

\section{Introduction}

Changes in charge density as a response to an external stimuli form basis for numerous applications. Knowing where the atoms are allows numerous deductions to be drawn solely on symmetry arguments. Symmetry essentially dictates what is possible, though typically it does not give absolute values for physical properties. An example is provided by ferroelectrics (Lines \& Glass, 1998). Ferroelectrics are a special case of pyroelectric materials, whose properties depend on the spatial scale considered. Single crystals are rather trivial case in which straightforward tensor formulation gives precise description of the physical properties. Obviously, knowledge of single crystal properties is not sufficient, as most materials utilized in applications are polycrystalline. Thus, one must understand how individual crystals are connected and how such a system responds to an external stimulus. This serves as a basis for dividing materials response to an intrinsic and extrinsic contributions. The former is essentially a single crystal response, whereas the latter takes into account the coupling between individual crystals and changes due to the phase transition induced by a stimuli. In practice, the stimuli are stress, heat or electric field. The importance of understanding the intrinsic and extrinsic contributions is not only related to the magnitude but also to the reversibility of the process. Piezoelectric actuators are based on the change in the charge density due to an external stress or change in dimensions by an applied electric field. Very challenging task is to produce a material yielding a reversible response, as the piezoelectric actuators used in atomic force microscopes demonstrate. Activities are ongoing to develop better materials for high precision devices (Hinterstein et al., 2011; Hoffmann \& Kungl, 2004). Another topical application is related to the piezoelectric energy harvesting in which a practical way of extracting energy is achievable through the Ericsson energy conversion cycle (Pruvost et al., 2010). Similarly, pyroelectric materials are utilized in infrared radiation detection matrices or pyroelectric energy harvesting components (Olsen \& Evans, 1983). In each case it is essential to understand the contribution of grain boundaries, ferroelectric domains within the grains, changes in fractions of different crystal species and the intrinsic contribution within an individual domain. As a further issue one must consider time-dependent phenomena, which reflect the fact that different contributions to polarization 
reversal dominate in different time-domains. An example where time-dependent phenomena are crucial is ferroelectric memory (Dawber et al., 2005).

Classical yet actively studied piezoelectric system is lead-zirconate titanate (PZT) (Jaffe et al., 1970). Though nearly all methods one can imagine have been applied to study the structural properties of PZT, the most widely used techniques are spectroscopic, $x$-ray and neutron powder diffraction, and transmission electron microscopy and electron diffraction (Glazer et al., 2004; Ricote et al., 1998). Since the early study of the lattice dynamics of lead titanate (Burns \& Scott, 1970), Raman scattering has been successfully applied to study the lattice dynamics of PZT based ceramics as a function of composition and temperature (Frantti et al., 1999a;b; Souza-Filho et al., 2002), stress (Ohno et al., 2006) or to address the domain configuration in thin films (Nishida et al., 2005). Understanding the domain formation is crucial when thin films are deposited. By a correct choice of single crystal substrate material and crystal cut one can deposit highly oriented thin films, though perfectly epitaxial films may not easily be achieved. Frequently deposition is carried out with in-situ substrate heating, which means that different domains are formed to relieve the stress due to the differences in the thermal expansion coefficient of the film and substrate, besides the usual straining or misfit dislocation formation (Kolasinski, 2008; Lüth, 2001). Raman spectroscopy has been notably useful for studying structural properties small particle size powders (Camargo et al., 2009) or fibres (Kozielski et al., 2010) as x-ray diffraction techniques often have limited ability to distinguish crystal symmetries of nanoscale structures. Even dielectric properties of nanosized powders can be accessed via the Lyddane-Sachs-Teller relation (Kano et al, 2007). In contrast to the powder diffraction techniques electron microscopy techniques have the advantage to pinpoint the area under study. The disadvantage (or at least an aspect to be kept in mind) is that sample thinning modifies the domain configuration of the sample, as is discussed below. Constructing a picture of PZT thus relies on data collected on very different type samples and techniques. Both phenomenological and first-principles modelling techniques are required to fulfill the missing data (experimentally not accessible) or to interpret the experimental results.

The present text aims to review symmetry based methods for initial stage structural studies. Combined crystallographical and thermodynamical methods prove to be very useful for understanding possible domain configurations in ferroelectric oxides. In this chapter basic piezo-, pyro-, and ferroelectric concepts are summarized, after which crystallographical aspects of domain formation in ferroelectrics are briefly summarized. The rest of the chapter focuses on the intrinsic and extrinsic contributions in PZT.

\section{Definitions}

\subsection{Crystal symmetry constraints for piezo-, pyro-, and ferroelectricity}

Piezoelectricity is exhibited in all but one (exception is the crystal class 432) noncentrosymmetric crystal class and is described by a third-rank tensor. Piezoelectric phenomena cover two cases, direct and converse effects, which, when matrix notation is used, are summarized by equations (see, e.g., (Newnham, 2005; Nye, 1995)) 


$$
\begin{aligned}
& P_{i}=d_{i j} X_{j} \text { direct effect } \\
& x_{i}=d_{i j} E_{j} \text { converse effect }
\end{aligned}
$$

in which $P_{i}$ is the polarization (in units $\mathrm{Cm}^{-2}$ ), $X_{j}$ is the applied stress using the Voigt notation (in units $\mathrm{Nm}^{-2}$ ), $x_{i}$ is the strain, $E_{j}$ is the applied electric field (in units $\mathrm{Vm}^{-1}$ ) and $d_{i j}$ are the piezoelectric constants (either in units of $\mathrm{CN}^{-1}$ or $_{\mathrm{mV}}^{-1}$ ). Thermodynamic consideration shows that the piezoelectric constants for direct and converse effects are the same (see, e.g., (Dove, 2003; Nye, 1995)). Fig. 1 illustrates the direct piezoelectric effect in the case of a tetragonal (space group $\mathrm{P} 4 \mathrm{~mm}$ ) $A B \mathrm{O}_{3}$ perovskite (Dove, 2003). Though crystals point groups symmetry (or crystal class) alone suffices to determine the number of piezoelectric constants, the practical situation is more involved as it is the whole sample, typically ceramic pellet or thin film, which gives the response to an applied stimulus. Further, the piezoelectric constants are not quite constants but do depend on temperature or the applied stimulus, such as applied electric field or stress. These issues are addressed in section 6.

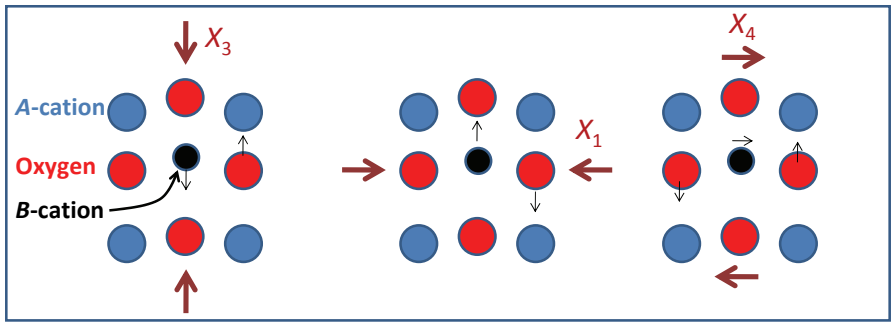

Fig. 1. Understanding the relationship between the crystal structure and property is crucial. Given example is for tetragonal (space group $\mathrm{P} 4 \mathrm{~mm}$ ) perovskite single crystal. Classical examples are $\mathrm{BaTiO}_{3}$ and $\mathrm{PbTiO}_{3}$. In the absence of external stimuli polarization appears along the $c$-axis. Application of stress $X_{1}, X_{3}$ and $X_{4}$ in turn results in the illustrated changes in polarization. Figure after M. Dove (Dove, 2003).

Figure 2 depicts pyroelectric and ferroelectric phenomena (Lines \& Glass, 1998). Pyroelectricity is a phenomenon found in crystals with a permanent electric dipole moment. The magnitude of this moment depends on temperature, which is the basis for experimental observation of the phenomenon. Symmetry consideration dictates that pyroelectricity can only occur in the ten crystal classes(Klapper \& Hahn, 2005): 6mm, 4mm, and their subgroups $6,4,3 m, 3, m m 2,2, m, 1$. All classes are piezoelectric, so the pyroelectric materials are a special case of piezoelectric materials. This also brings an experimental difficulty concerning the practical determination of the possible pyroelectricity of the crystal: within the crystal temperature changes inhomogeneously, which causes strains. Strains in turn add piezoelectric contribution to the measured electric charges. This means that often one can only conclude that the studied crystal lacks a centre of symmetry. Ferroelectric crystals are those pyroelectric materials in which the direction of the permanent electric dipole moment can be changed by an electric field. 
(a)

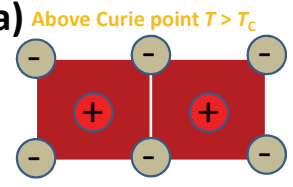

Below Curie point $T<T_{\mathrm{C}}$

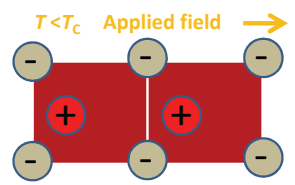

(b)
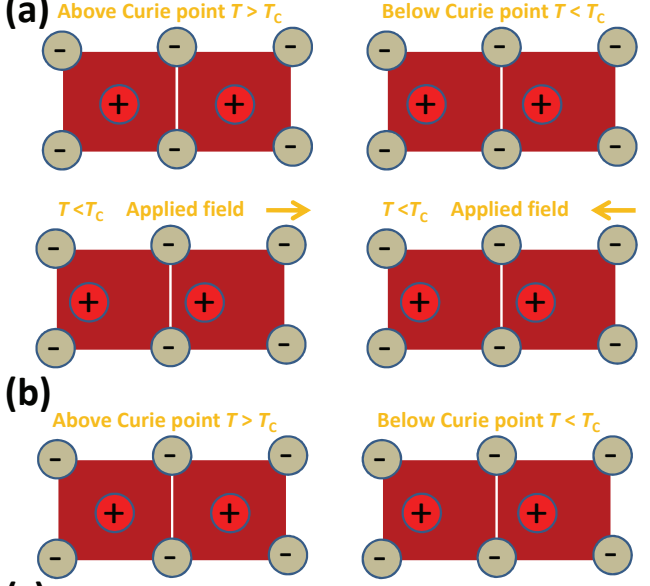

(c)

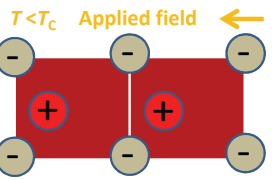

Fig. 2. Schematic illustration of the (a) pyroelectricity and (b) ferroelectricity. Figure after Lines and Glass(Lines \& Glass, 1998).

\section{Twins and ferroelectric domains}

Theory of twinning is rich and consequently the terminology used in literature is sometimes ambiguous. In the following brief summary of twinning we adopt the definitions given in refs. (Hahn \& Klapper, 2003; Koch, 2004) according to which an intergrowth of two or more macroscopic, congruent or enantiomorphic, individuals of the same crystal species is called a twin, if the orientation relations between the individuals occur frequently and are crystallographic. The individuals are called twin components or (twin) domains.

3.0.0.1 Twin law and twin elements.

Twin domains are brought into parallel orientation with each other by a symmetry operation, the twin operation. Twin operation may occur only once in a macroscopic scale, and cannot be a symmetry operation of either of the twin domains.

Twins can be classified by their origin (for example, growth twins and transformation twins) or from crystallographical viewpoint. For ferroelectric domain formation, transformation twins are central. Crystallographically important transformation twins can be classified as merohedral and pseudo-merohedral twins.

\subsection{Transformation twins}

Transformation twins are formed when a phase transition between the prototype phase, virtual or real (space group $G$ ) and a lower symmetry phase (space group $H$ ) takes place. 
By further assuming that the two phases posses a group-subgroup relationship crucial information about twin domain formation can be extracted from the International Tables for Crystallography, Volume A (ITCA)(Hahn, 2005), which tabulates maximal non-isomorphic subgroups $H$, Type I: translationengleiche $(t)$ subgroups in which all translations are retained, Types IIa and IIb: klassengleiche ( $k$ ) subgroups, which are obtained by 'decentring' the conventional cell and enlarging the conventional cell, respectively. Thus, not all translations are retained. Three cases can now be distinguished (Hahn \& Klapper, 2003): (1) $H$ is a $t$ subgroup of $G,(2) H$ is a $k$ subgroup of $G$, (3) Cases 1 and 2 occur together ( $H$ is a general subgroup of $G$ ). Transformation twins provide a close link to the Landau's phase transition theory. The equivalent solutions for the order parameter are represented by different twin domains.

\subsection{Merohedral twins}

Merohedral (non-ferroelastic) twinning corresponds to the case where the twin-element is an element of the crystal system (the crystal class of the lattice) but not an element of the point group of the crystal (crystal class). Characteristic feature of the merohedral twinning is that the lattices of all twin domains coincide exactly. The merohedral twinning corresponds to non-ferroelastic phase transition and ferroelectric domains can be formed, as the example of lithium niobate $\left(\mathrm{LiNbO}_{3}\right)$ demonstrates.

3.2.0.2 Example: domains in lithium niobate.

At elevated temperatures (transition is reported to occur between 1323 and $1473 \mathrm{~K}$, depending on the sample stoichiometry) $\mathrm{LiNbO}_{3}$ has the high-symmetry phase $R \overline{3} c$, which transforms via nearly second order transition to ferroelectric $R 3 c$ phase, so that an inversion is lost in the transition. The transition is classified as an order-disorder type with a displacive component (Boysen \& Altorfer, 1994; Hsu et al., 1997). No change in crystal system occurs, the twin element being an inversion element. The transition is non-ferroelastic ferroelectric in which $180^{\circ}$ domains are formed. Thus, the possible domains are limited by crystallographical considerations, though domain boundaries are characteristically rounded.

\subsection{Pseudo-merohedral twins}

An important special case of pseudo-merohedral twins are twins formed in ferroelastic phase transition. In the following we consider a phase transition from the prototype phase to a ferroelastic and ferroelectric phase possessing a group-subgroup relationship though the transition is not necessarily continuous. However, it is necessary that the crystal system is changed in the transition. From the metrical viewpoint the differences in lattice parameters between the high- and low-symmetry phases are small. The structural symmetries lost in the phase transition are preserved as pseudosymmetries (Hahn \& Klapper, 2003; Strukov \& Levanyuk, 1998). We further assume that the transition is displacive so that the atoms in the lower symmetry phase are slightly shifted from their high-symmetry positions. This covers technologically important ferroelastic-ferroelectrics, such as Rochelle salt and $\mathrm{BaTiO}_{3}$.

3.3.0.3 Example: domain formation in tetragonal and rhombohedral lead zirconate titanate.

Lead zirconate titanate $\left[\mathrm{Pb}\left(\mathrm{Zr}_{x} \mathrm{Ti}_{1-x}\right) \mathrm{O}_{3}, \mathrm{PZT}\right]$ possess a cubic perovskite crystal structure (space group $P m \overline{3} m$ ) above the Curie temperature (increasing from about $520 \mathrm{~K}$ to $765 \mathrm{~K}$ 
with increasing Ti content). Titanium rich PZT (up to roughly $x=0.50$ ) transforms via first order transition to the tetragonal, space group P4mm, phase. When $1<x<0.55$ the cubic phase transforms to rhombohedral $R 3 m$ phase via (nearly) second-order transition. Following (Hahn \& Klapper, 2003) the transitions are theoretically divided into two steps, both involving a transition to a maximal non-isomorphic subgroup. First is a ferroelastic, non-ferroelectric transition:

Ti-rich PZT $P m \overline{3} m \rightarrow P 4 / m m m$ ( $t$ transition)

Zr-rich PZT $P m \overline{3} m \rightarrow R \overline{3} m$ ( $t$ transition)

Transition to the $P 4 / \mathrm{mmm}$ phase corresponds to the loss of threefold rotation axes (the list of elements preserved in the transition are listed in the ITCA), which results in three spontaneously strained (nearly $90^{\circ}$ ) twin domains in each which the tetragonal $c$-axis points towards one of the three former cubic $a, b$ and $c$ axes. The lattice distortion is small, so the aforementioned condition for pseudo-merohedral twinning is fulfilled. Transition to the $R \overline{3} m$ phase means a loss of fourfold axes, fourfold axes with centre of symmetry, $\{100\}$ mirror planes, leaves inversion centre, one pair of threefold rotation axis, one pair of threefold rotation with centre of symmetry and three twofold rotation axes. This leads to four ferroelastic $70.5^{\circ}$ twin domains related by the lost (cubic) $\{100\}$ mirror planes. All domains $(P 4 / \mathrm{mmm}$ and $R \overline{3} \mathrm{~m})$ are centrosymmetric.

The second transition is between the ferroelastic and ferroelectric phases and involves ionic displacements resulting in the loss of inversion:

Zr-rich PZT P4/mmm $\rightarrow$ P4mm ( $t$ transition)

Ti-rich PZT $R \overline{3} m \rightarrow R 3 m$ ( $t$ transition)

Each twin domain splits into two antiparallel polar ferroelectric $180^{\circ}$ domains.

\subsubsection{Two-phase systems.}

Numerous studies have been conducted to understand the behaviour of morphotropic phase boundary (MPB) lead zirconate titanate. After a monoclinic $\mathrm{Cm}$ phase was reported to exists in PZT within the MPB region (Noheda et al., 1999; 2000) many studies were dedicated to understand the electromechanical properties through the crystallographical properties of the single $\mathrm{Cm}$ phase. However, it turned out that the $\mathrm{Cm}$ phase co-exists with the rhombohedral R3c phase (Frantti et al., 2002; Yokota, 2009). There are many reports which use the Cc symmetry, instead of the $R 3 c$ symmetry. It has been very difficult to prepare single crystal PZT and most of the studies focused on either ceramic pellet or on powder samples. The symmetry choice has been very controversial topic (see, e.g., review (Frantti, 2008b) and references therein) and it was not until very recently that a detailed neutron difraction study on PZT single crystals could solve the issue by providing data which ruled out the $C c$ symmetry (Phelan et al., 2010). The study found that the $R 3 m / R 3 c$ and $C m$ domains coexist. This is important finding as it provides an explanation to the exceptionally good piezoelectric properties of PZT ceramics, which is discussed in section 7.2. As the results given in ref. (Yokota, 2009) reveal, the two-phase co-existence is extended towards large Zr-contents so that there is no clear phase boundary. In the first-order phase transition both phases co-exists, though it is not obvious why the $\mathrm{Cm}$ phase (slightly distorted version of the tetragonal $\mathrm{P} 4 \mathrm{~mm}$ phase) is found in large composition range, extending from $x=0.52$ up to 0.92 (Frantti et al., 2002; Noheda et al., 1999; 2000; Yokota, 2009). The reason for the distortion is not clear, though it seems important to consider the contact relationships between the two phases (intergrowth of the rhombohedral and monoclinic crystals). 


\section{Mechanical and electrical compatibility conditions}

In practice, ferroelectrics show small number of domain boundary types, which can be understood by considering mechanical and electrical compatibility conditions. In the case of mechanical boundary condition a simple method is to consider the strain on the crystals separated by the twin domain boundary (given, e.g., in ref. (Hahn \& Klapper, 2003)) and to look for stress-free contact planes. Mechanical compatibility condition means that the boundaries minimizing the strain energy are favored.

Figure 3 shows charged and non-charged $180^{\circ}$ merohedral and $90^{\circ}$ zigzag domain boundary possessing large domain wall energy (Hahn \& Klapper, 2003), and a method to introduce $180^{\circ}$ tail-to-tail (TT) and head-to-head (HH) domains by providing charge compensation through acceptor and donor layers (Wu \& Vanderbilt, 2006). In ferroelectrics one must also consider the contribution of electrical charges at the boundaries. Basically charge neutral boundaries are energetically favored, expressed by the condition $P_{n}(2)-P_{n}(1)=\rho=0$, where $P_{n}(1)$ and $P_{n}(2)$ are the components of the spontaneous polarization normal to the boundary of the two domains and $\rho$ is the charge density. The situation can be different in conducting (either in prototype phase or ferroelectric phase) samples due to the ferroelectric polarization compensated by free charge carriers. In practice in insulating materials $\mathrm{HH}$ and TT domains are ruled out as the electrostatic energy makes them unfavorable. Thus, the merohedral twins are characteristically $180^{\circ}$ domains with interfaces parallel to the polarization axes. It was proposed, based on the first-principles calculations, that $\mathrm{HH}$ and TT domains could be stabilized by inserting donor and acceptor layers to compensate the charge otherwise formed in the domain wall (Wu \& Vanderbilt, 2006). As a case study $\mathrm{PbTiO}_{3}$ based ferroelectric 8-cell superlattice with $\mathrm{Sc}^{3+} \mathrm{O}_{2}$ (acceptor layer) and $\mathrm{Nb}^{5+} \mathrm{O}_{2}$ (donor layer) was studied, with the prediction that such a structure would be stable with a ground state symmetry Pmm2. Analysis of local polarization revealed that the superlattice is antiferroelectric in the [001] direction, and ferroelectric in the [100] direction.

\subsection{Why to worry about domains}

\subsubsection{Ferroelectric hysteresis}

Measuring the polarization as a function of electric field, i.e., hysteresis loop, measures the energy required to twice reverse the polarization direction. In ceramics a large fraction of the energy necessary for polarization reversal is consumed to domain wall motion. Depending on the applications one deliberately modifies the composition so that the coercive field is either large (hard ferroelectric) or small (soft ferroelectric) material. In PZT this is achieved by controlling the domain wall motion by doping with higher- or lower-valent ions (Jaffe et al., 1970). Controlling the amount and position in the ceramics is thus crucial: it makes a difference if the dopants are segregated in the grain boundaries or if they are homogeneously distributed. Useful models and practical examples of spatial distribution of dopant atoms in polycrystalline materials are provided in ref. (Phillips, 2001). Fig. 4 shows a hysteresis loop, plotting polarization versus applied field $E$, characteristic to a soft ferroelectric material as seen from the low value of the coercive field $E_{C}$. In the case of hard ferroelectric material the coercive field is larger and the shape of the loop is more rectangular. Characteristically hysteresis is measured at quasi-static conditions, which implies that the $E_{C}$ value is determined by the polarization reversal mechanisms dominant in the static limit. The mechanism do depend on the timescale in concern and also on the sample geometry. 
(a)

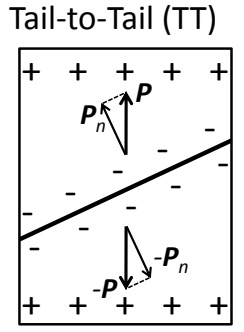

(b)

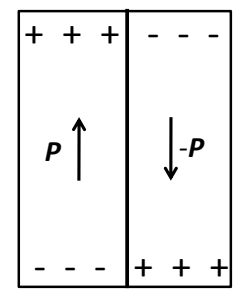

(c) $\mathrm{H}$

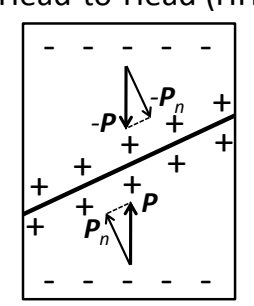

(d)

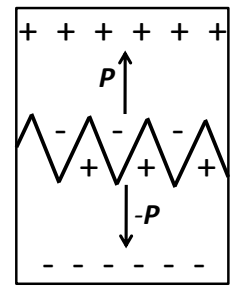

(e)

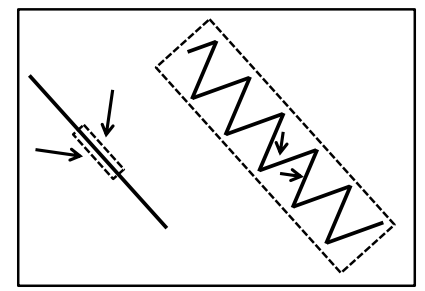

(f)

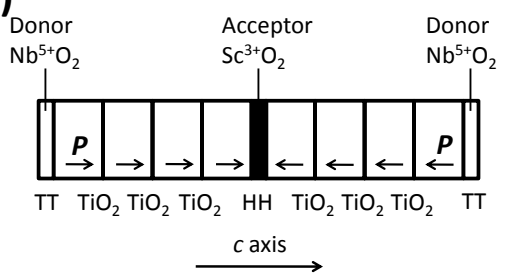

Fig. 3. (a) Tail-to-tail, (b) uncharged, (c) head-to-head and (d) charged zigzag $180^{\circ}$ merohedral domain boundaries. The boundary (b) is also common among pseudo-merohedral domain boundaries. Figures (a) to (d) after ref. (Hahn \& Klapper, 2003). Panel (e) shows the $90^{\circ}$ pseudo-merohedral domain boundary which at first glance appears as a head-to-head domain boundary, but in a detailed study turned out to be a zigzag boundary where all boundaries are head-to-tail, shown in the right-hand corner. Figure after ref. (Lines \& Glass, 1998; Yakunin et al, 1972). Panel (f) shows a ferroelectric superlattices used in a first-principles computational study in which head-to-head and tail-to-tail domain boundaries are stabilized by placing donor and acceptor layers to serve as charge compensation layers (Wu \& Vanderbilt, 2006).

The first aspect is discussed in section 4.1 .3 by reviewing very recent studies, whereas the latter obviously involves depolarization field and phenomena related to the substrate and thin-film interactions. Technologically important case is a thin film capacitor, in which case the depolarization field significantly affects the $E_{C}$ value. A finite-element study of the topic is given in ref. (Pane et al., 2008).

\subsubsection{Irreversibility of domain switching in PZT.}

The equations describing the piezoelectric responses (section 2) are linear, which is usually a valid approximation. There are, however, cases in which the linearity assumption ceases to be accurate. The most obvious is the vicinity of the phase transition which may result in large changes in piezoelectric constants (Frantti et al., 2007), which is an intrinsic contribution. This means that when a phase is about to change to another phase or orientation state (domain switching) external stimulus (temperature, stress, electric field) can cause large changes in the materials response (strain or induced charge). Many applications, such as actuators required to operate in a precise and repeatable manner, require that the change is reversible, which is not obvious. One may recall that many piezoelectric solid-solutions, PZT being a textbook example, have a composition adjusted so that two competing phases, tetragonal 


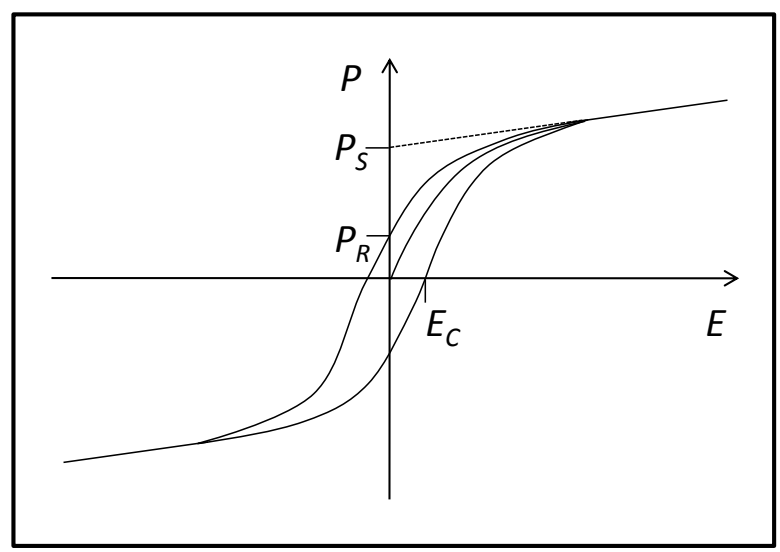

Fig. 4. Schematic illustration of the hysteresis loop with characteristic values indicated: coercive field $E_{C}$, remanent polarization $P_{R}$ and $P_{S}$, taken as the saturation value of the polarization extrapolated to zero field.

(or the $\mathrm{Cm}$ phase, which can be considered as pseudotetragonal for most of the purposes) and rhombohedral, are energetically almost as favorable. By symmetry, the transition from tetragonal to rhombohedral phase must be of first order, which implies that there is a hysteresis related to the transition. This hysteresis means that the response of the system depends on the history, so that the response to heating and cooling are frequently different. Also extrinsic contribution results in non-linearities.

The quasi-static (a cyclic driving electric field was applied during the diffraction experiment using unipolar and bipolar square waves at $1 \mathrm{~Hz}$ and amplitudes equal to or below the coercive field) time-dependent studies carried out by stroboscobic neutron and x-ray powder diffraction data collection technique on pure and La- or Fe-doped PZT samples with composition in the vicinity of the morphotropic phase boundary, show that already below coercive field non-linear terms become significant, which was related to the non- $180^{\circ}$ domain switching(Jones et al., 2006; Pramanick et al., 2009a;b).

\subsubsection{Kittel's law and domains in thin films}

An example where the size, shape and sample preparation method affect ferroelectric domain formation are thin films (either free-standing or deposited on a substrate) or samples prepared for transmission electron microscopy (TEM) studies. An insulating ferroelectric materials splits into several domains to minimize the energy of the crystal geometry dependent depolarization electric fields due to the uncompensated dipoles on the surface (assuming that there are no free charges available or at least that the domain formation occurs before the compensation takes place). In the simplest model the splitting of materials into domains oriented so that their net polarization is zero is limited by the cost due to domain wall creation. Thus, in the simplest terms one has, in addition to the usual polynomial order parameter expansion two other contributions to the ferroelectric free energy: depolarization field and domain wall energy. An illustrative example is provided by the model for $180^{\circ}$ domains in which case one can derive so called Kittel's law according to which the width of the domains 
is proportional to the square root of the thickness. In his original work Kittel considered ferromagnetic materials(Kittel, 1946). Analogous treatment was applied to ferroelectric materials by Mitsui and Furuichi (Mitsui \& Furuichi, 1953) and to ferroelastic materials by Roytburd (Roitburd, 1976) and were summarized in ref. (Schilling et al., 2006). Following ref. (Lines \& Glass, 1998) the essential result is summarized by the equation $d=\left(\frac{\sigma t}{\varepsilon^{*} P_{0}^{2}}\right)^{1 / 2}$, where $d$ is the domain width, $t$ the domain thickness, $P_{0}$ is the polarization at the center of a domain, $\sigma$ is the domain wall energy per unit area and $\varepsilon^{*}$ is a constant depending on the dielectric constants of the ferroelectric. Other expressions for the Kittel's law consider the influence of substrate, which can be significant and change the proportionality constant, are discussed in ref. (Streiffer et al., 2002). The proportionality $d \propto t^{1 / 2}$, however, remains. Similar relationship between domain width and thickness, but with different proportionality constants, is found for $90^{\circ}$ domains in epitaxial ferroelectric and ferroelastic films (Pertsev \& Zembilgotov, 1995). A TEM study conducted on free-standing lamellae showed that when the thickness gradient is perpendicular to the domain walls the domain width continuously decreases with decreasing thickness, following the Kittel's law (Schilling et al., 2006). The study found two other mechanisms occurring in thin lamellae: bifurcation in domains parallel to the thickness gradient and discrete period changes at the interface between clusters of stripes perpendicular to each other. As the domain wall motion and nucleation of new domains are important for polarization reversal, the thickness and geometry dependent factors are central for designing thin film components, such as ferroelectric memory cells. For fundamental research it is evidently crucial to understand the domain formation in bulk materials (say, used in many neutron powder diffraction studies) and in thin films prepared for TEM studies in order to avoid wrong conclusions.

\subsubsection{Time-dependent studies of polarization reversal in PZT}

Polarization reversal may involve the growth of existing domains, domain-wall motion or the nucleation and growth (either along the polar direction or by sideways motion of $180^{\circ}$ ) of new antiparallel domains (Dawber et al., 2005; Lines \& Glass, 1998). The mechanism dominating depends on the material, applied field and electrode type, sample geometry and time domain. In ferroelectrics polarization reversal is typically modelled to be inhomogeneous, where nuclei of domains with polarization parallel to the applied field initially form at the electrodes, grow forward direction (typically considered to be fast process, addressed below) and then grow by sideways motion (slow in perovskite oxides)(Dawber et al., 2005).

In low-frequency experiments the breakdown field is at around 100 and $200 \mathrm{MV} / \mathrm{m}$. Breakdown, however, is not an instantaneous phenomenon, and thus for a short times one can apply much larger fields than breakdown field. Recently, experimental studies of short-time structural changes in ferroelectric thin films became accessible through x-ray synchrotron instruments. Time-dependent phenomena, notably the nonlinear effects in the coupling of polarization with elastic strain and the initial stage of polarization switching were addressed in refs. (Grigoriev et al., 2008; 2009). In these studies capacitors containing $35 \mathrm{~nm}$ thick epitaxial $\mathrm{Pb}\left(\mathrm{Zr}_{0.20} \mathrm{Ti}_{0.80}\right) \mathrm{O}_{3}$ ferroelectric thin films were studied by time-resolved $\mathrm{x}$-ray microdiffraction technique in which high-electric field (up to several hundred $\mathrm{MV} / \mathrm{m}$ ) pulses were synchronized to the synchrotron x-ray pulses. Demonstration of the capability of the technique is an experiment where $8 \mathrm{~ns}$ long electrical pulses of $24.4 \mathrm{~V}$ were applied to the PZT capacitor, yielding $2.7 \%$ strain, record among piezoelectric strains (year 2008) 
(Grigoriev et al., 2008). The same study revealed that the piezoelectric $d_{33}$ coefficient only slightly increases when the applied field increases to $160 \mathrm{MV} / \mathrm{m}$, whereas more strong increase occurs above $180 \mathrm{MV} / \mathrm{m}$ so that the $d_{33}$ coefficient has the low-field value at 395 $\mathrm{MV} / \mathrm{m}$. The increased $d_{33}$ coefficient value between 180 and $395 \mathrm{MV} / \mathrm{m}$ was assigned to the Ti-O bond elongation (Grigoriev et al., 2008). Another technologically relevant finding was related to the initial stage of polarization switching: a series of $50 \mathrm{~ns}$ duration pulses did not switch the polarization if the field was below $150 \mathrm{MV} / \mathrm{m}$, even when the total pulse duration was several milliseconds (Grigoriev et al., 2009). It is also worth to note that 150 $\mathrm{MV} / \mathrm{m}$ was estimated to be three times the low-frequency $E_{C}$ and in low-frequency hysteresis measurements $1 \mathrm{~ms}$ above the $E_{C}$ is sufficient for polarization reversal. To address the stability of unswitched polarization states three possible explanations were considered: (i) slow initial domain propagation, limited by the time required for the establishment of charge distribution necessary for the movement of curved (charged) domain walls (Landauer, 1957), (ii) disappearance of small domains between pulses and (iii) nucleation times are longer than $50 \mathrm{~ns}$ applied in the study (Grigoriev et al., 2009). The first and third explanation were found plausible, whereas the second explanation was ruled out as it was estimated that 50 ns is sufficient for a nucleated domain to reach stable size (which can be estimated through thermodynamical considerations, see ref. (Strukov \& Levanyuk, 1998)).

\section{Intrinsic and extrinsic contributions}

Terms intrinsic and extrinsic contribution are commonly used in literature. Though both contributions frequently occur simultaneously, it is helpful to trace the origin of the piezoelectric response down to atomic scale. Piezoelectric materials response involves changes in the primitive cell level and also in the larger scale, in which case the motion of domain boundaries and grain boundaries must be taken into account. Fig. 5 illustrates a polycrystalline material consisted of grains, which in turn contain domains. The applied stimulus is transmitted via grains, and results in changes in grain boundaries and domain wall motion. Both are examples of an extrinsic contribution. The stimulus also causes changes within a primitive cell, an example is the shift of an oxygen octahedra with respect to A-cations in perovskites, Fig. 1. The structure of the sample structure significantly influences its response to an external stimulus, examples being poled polycrystalline ceramics and non-twinned single crystals. Correct treatment of piezoelectric response requires the determination of the texture present in the sample as it is the whole system, consisted of variously oriented domains (or crystallographical twins), which responds to an external stimulus. After the texture, or preferred orientation, is known appropriate angular averages of piezoelectric constant can be determined. For instance, electrically poled ceramics belong to symmetry group $\infty m$ (Newnham, 2005). Texture, and individual piezoelectric constants, change as sufficiently large stimuli are applied. This section summarizes the changes occurring in the atomic scale in piezoelectric materials by dividing the response to changes occurring in the individual primitive cells and changes occurring in the domain distribution.

\section{Intrinsic contribution}

By intrinsic term one refers to the changes in electric polarization within a domain as a response to an external stimulus. This implies that no domain wall motion or changes in 


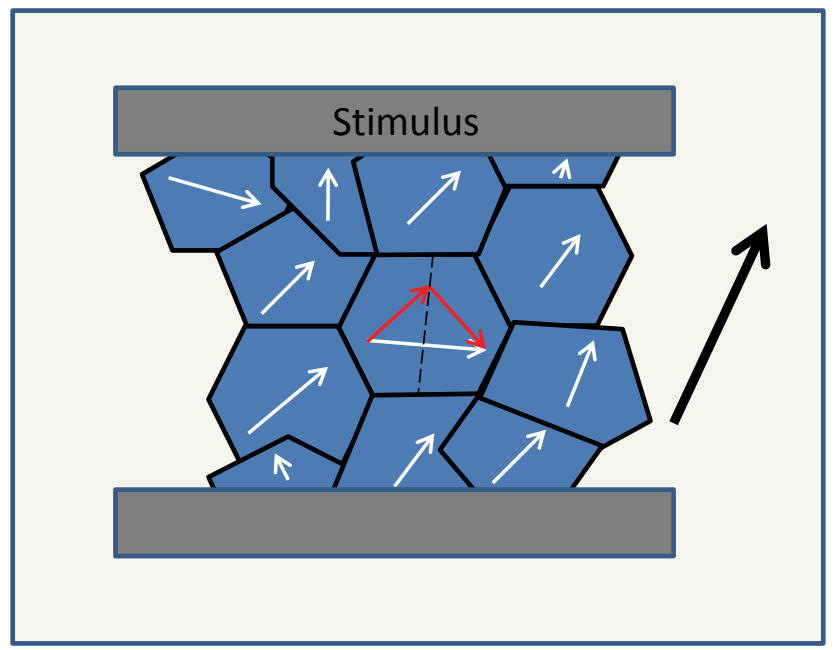

Fig. 5. Schematic illustration of different contributions resulting in the net polarization (black arrow). When stress is applied to a polycrystalline material, the effect is transmitted via grains (polygons). Each grain in turn is divided into domains, exemplified by a $90^{\circ}$ domain wall (dashed line). Red arrows indicate the polarization directions within the domains, and the white arrow is the resultant polarization.

phase fraction is taken into account. An example is given in Fig. 1 in which the piezoelectric response of a $A B \mathrm{O}_{3}$ perovskite is shown for different applied stress. As a special case of an intrinsic response is the $180^{\circ}$ domain reversal. It is also worth noting that applied stimuli frequently breaks the equilibrium symmetry, though the symmetry changes may not always be experimentally resolved. Computationally the piezoelectric and elastic constants can be determined by fist-principles techniques, which is a very useful method for estimating the pure intrinsic contribution. Notable care should be paid on the phase stability, as computation of the crystal properties of unstable phases results in meaningless results. In the context of pressure induced transitions in $\mathrm{PbTiO}_{3}$ the phase stability issues were addressed in refs. (Frantti et al., 2007; 2008a). Recent inelastic neutron scattering study suggests that a phase instability induced by a polar nanoregion-phonon interaction contributes to the ultrahigh piezoelectric response of $\mathrm{Pb}\left(\mathrm{Zn}_{1 / 3} \mathrm{Nb}_{2 / 3}\right) \mathrm{O}_{3}-4.5 \% \mathrm{PbTiO}_{3}$ and related relaxor ferroelectric materials (Xu et al., 2008). Presently an ab-initio computational modelling of a PZT solid-solution is a formidable task as it would require enormous supercells. One way to bypass this problems is to mimic the 'chemical pressure' (partial substitution of Ti by $\mathrm{Zr}$ ) by hydrostatic pressure. Density-functional theory (DFT) computations predict that at $0 \mathrm{~K}$ (ground state) a phase transition between tetragonal $\mathrm{P} 4 \mathrm{~mm}$ and rhombohedral $R 3 \mathrm{c}$ phase take place at 9.5 GPa pressure (Frantti et al., 2007), which suggests that some insight about the PZT system can be drawn. Significant increase of certain piezoelectric constants, notably the $d_{15}$, was observed once the phase transition was approached. Thus, the vicinity of the phase transition causes that also intrinsic contribution is significantly increased. Experimental and computational studies suggest that the curvature of the phase boundary is determined by two factors, the entropy term favouring the tetragonal phase, and the oxygen octahedral tilting 
giving an advantage for the rhombohedral $R 3 c$ phase (Frantti et al., 2009). Octahedral tilting, characteristic to the $R 3 \mathrm{c}$ phase, allows efficient volume compression (Thomas \& Beitollahi, 1994).

In thin films biaxial stress can be used to tune the piezoelectric properties by deliberately straining the material, in which case strain engineering is a term used for a thin film technology method applied to improve the piezoelectric properties (Janolin, 2009). The phase diagram in thin films is often quite different from the one found for bulk ceramics, which in turn may result in significant differences in electromechanical response (Janolin, 2009; Liu et al., 2010). The interplay between film thickness and different stress has a large impact on stress relaxation mechanism (Janolin, 2009; Liu et al., 2010).

\subsection{Notes on polarization rotation model}

There have been attempts to explain the piezoelectric response of many perovskite solid solution systems in the vicinity of the morphotropic phase boundary through (more or less) continuous polarization rotation. Characteristically, the morphotropic phase boundary separates tetragonal and rhombohedral phases. The common feature of these models is that focus is put on the intrinsic part of the piezoelectric response, specifically on the rotation of polarization vector between the tetragonal polarization direction, $\langle 001\rangle$, and rhombohedral polarization direction, $\langle 111\rangle$, and the extrinsic contributions are simply discarded. This type of transition route was essentially based on the computational study on monodomain $\mathrm{BaTiO}_{3}$ according to which it takes less energy to rotate the polarization along the 110 plane than through path which is consisted of segments parallel to the unit cell edges(Fu \& Cohen, 2000), which was commonly believed to explain the high electromechanical response observed in many perovskite oxide solid-solutions. However, the transition between the tetragonal and rhombohedral phases is necessarily of first order, implying hysteretic transition in which the phase proportions between the two phases varies as a function of composition.

The small but unambiguous monoclinic distortion (space group $\mathrm{Cm}$, monoclinic $c$-axis is deviated by a less than half degree from the tetragonal $c$-axis, in contrast to the $55^{\circ}$ required to have a continuous rotation) observed in lead-zirconate-titanate ceramics within the MPB (Frantti et al., 2000; Noheda et al., 1999) and Zr-rich area(Yokota, 2009) suggests that one should consider the role of the $\mathrm{Cm}$ phase for the electromechanic properties. Though some reports in rather straightforward manner linked the exceptional electromechanical properties of lead-based piezoelectrics, such as PZT, to be due to the monoclinic distortion(s) serving as a bridging phase(s) between the rhombohedral and tetragonal phases (see also discussion ref. (Frantti et al., 2008a)), the following points should be noted:

- even though the polarization direction of the $\mathrm{Cm}$ phase can point in any direction in the mirror plane (as far as crystal symmetry is considered), experiments reveal that the monoclinic $\beta$ angle remains roughly constant through the whole composition area, being about $90.5^{\circ}$ : if there would be a continuous rotation from tetragonal to rhombohedral direction, it would be easily seen by standard diffraction techniques. However, no evidence for that is reported.

- the treatment given in ref. (Sergienko et al., 2002) shows that the phase transition between monoclinic and tetragonal phases can be of second order, the transition between rhombohedral and monoclinic phases must be of first order. This is consistent with the 
observed two-phase co-existence of $R 3 c$ and $C m$ phases (Frantti et al., 2002; Yokota, 2009): i.e., the $\mathrm{Cm}$ phase is not observed alone.

Our interpretation is that the monoclinic phase is not stable alone, but is probably due to the interaction between rhombohedral and tetragonal phases. In this spirit, it is worth to experimentally look the crystal boundary between rhombohedral and monoclinic phase. Certain external stimuli (e.g., $X_{1}$ and $X_{4}$ ) break the tetragonal symmetry, the magnitude of which can be estimated from the elastic constants. Thus, even internal stresses are able to lower the symmetry and the significance of the monoclinic distortion might be a stress relief, as was suggested in ref. (Topolov \& Turik, 2001)

We note that there are computational and experimental reports on $\mathrm{PbTiO}_{3}$ according to which hydrostatic pressure would induce monoclinic phase(s) intermediating the $P 4 \mathrm{~mm}$ and $R 3 m$ phases. However, it turned out that the computational study was carried out for an unstable phase (as could be revealed by enthalpy values and phonon instabilities) and the experimental data was interpreted in terms of a wrong structural model (the model Bragg reflection positions and peak intensities did not match with the experimental data). For more details, see refs. (Frantti et al., 2007) and (Frantti et al., 2008a). It is the opinion of the authors that after the intrinsic and extrinsic contributions are properly taken into account, an accurate and sufficient description for piezoelectricity is achieved.

\section{Extrinsic contribution}

Modeling extrinsic contribution is challenging, as it requires a description for domain boundary motion, which itself is rather complex process, and also a model for changes in phase fractions. Below a crystal boundary motion in an intergrowth and domain switching are discussed.

\subsection{Changes in phase fractions}

Studies of materials operating in the vicinity of the first-order phase transition require notable care as even small quantities of energy (e.g., in the form of heat or due to an applied field) can cause significant changes in the phase fractions. This is evidenced in PZT powders with composition at the MPB region by phase fraction changes in (pseudo-)tetragonal and rhombohedral phases as a function of temperature (Frantti et al., 2003). The two-phase co-existence is found in the Zr-rich side of MPB (Yokota, 2009), consistently with ref. (Sergienko et al., 2002) according to which the phase transition between monoclinic and rhombohedral phase is of first-order. The mechanism behind transformation is not yet clear, but it is probable that there are regions in which rhombohedral and monoclinic crystals are grown together. The phase transformation mechanism is crucial for the understanding of the piezoelectric response of PZT. Detailed studies to understand the atomic scale structure of the contact plane separating the two phases within the intergrowth and the movement of the plane under external stimuli are yet missing.

Fig. 6 shows an intergrowth of rhombohedral and monoclinic crystals. Though the reality is more complex, this type of intergrowth, and the contact plane motion, are suggested to have a crucial role for electromechanical response. Analogously to the domain boundary motion, phase transition (and changes in phase fractions) result in once the boundary moves. Needless to say, Fig. 6 does not imply continuous rotation, in contrast experiments indicate 


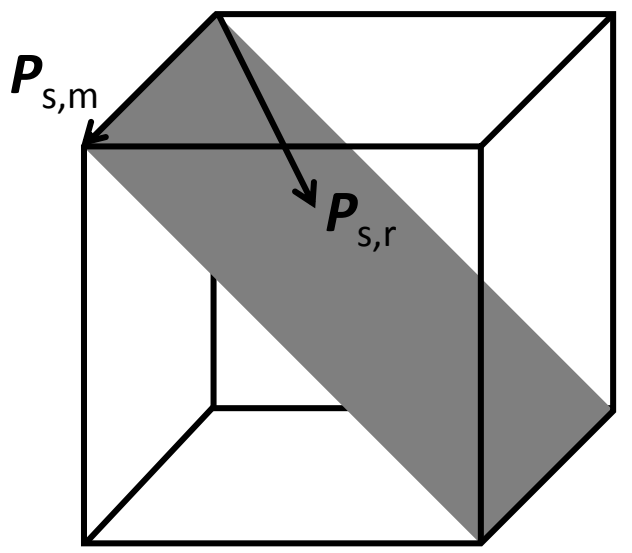

Fig. 6. Schematic illustration of an intergrowth of rhombohedral cystal (below the gray plane) and monoclinic crystal (above the gray plane). The directions of the spontaneous polarization in the monoclinic and rhombohedral phases are labelled as $\boldsymbol{P}_{s, m}$ and $\boldsymbol{P}_{s . r}$. Motion of the gray plane by external stimuli corresponds to a change in the phase fractions and would lead to large electromechanical response. No continuous polarization is involved.

discontinuous change. The electric and mechanical boundary conditions should serve as reasonable limiting factors when possible intergrowths are considered. Experimental studies are challenged by the fact that sample thinning influences the samples domain and grain boundary structure, implying that it is not straightforward to compare the results obtained from transmission electron microscopy technique and neutron powder diffraction studies (see section 4.1.2).

\subsection{Domain switching}

Figure 7 shows schematically the importance of a domain boundary. The lattice points of both domains are common at the boundary (implying no strain, so that the mechanical boundary condition is fulfilled), and since the polarization component perpendicular to the boundary does not change, also electrical compatibility requirement is fulfilled. However, the atom positions corresponding to the different domains at the boundary do not overlap: the atoms at the boundary region are disordered. One expects that also the polarization changes gradually in the domain wall, as is discussed in section 7.2.0.1. By applying external stimulus (e.g., stress) one domain state is preferred. In the simplest (perhaps excessively simple) picture the domain boundary sweeps through the energetically unfavorable domain. Now, there is an energy barrier for moving the atoms in the boundary zone. Obviously, if the difference between the atomic positions (shown in the upper part in Fig. 7(a)) is not large, switching is easy. Fig. 7(b) shows one way to diminish the stress in domain boundary by introducing a centrosymmetric cubic layer. The strain changes once one moves from the interior of the domain through the domain boundary, implying elastic energy which one must overcome in order to move the domain boundary 
A study about the domain switching showed that the $90^{\circ}$ domains in single phase tetragonal phase (titanium rich PZT) hardly switch, whereas the domains in the two-phase region switch (Li et al., 2005). Texture and strain analysis of the ferroelastic behavior of $\mathrm{Pb}\left(\mathrm{Zr}_{0.49} \mathrm{Ti}_{0.51}\right)_{3}$ by in situ neutron diffraction technique showed that the rhombohedral phase plays a significant role in the macroscopic electromechanical behavior of this material (Rogan et al., 2003). Figure
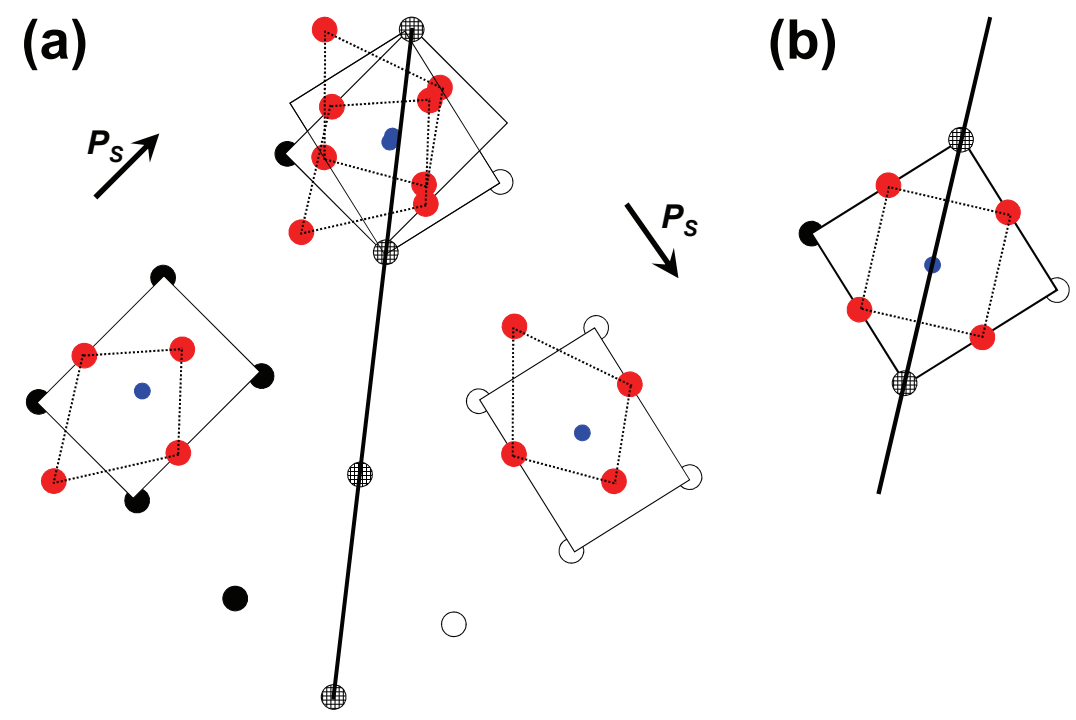

Fig. 7. (a) Schematic illustration of the (nearly) $90^{\circ}$ domains in tetragonal $A B \mathrm{O}_{3}$ perovskite. Continuous bold line shows the domain boundary. Black and unfilled spheres illustrate the lattice points of the different domains. The domain boundary lattice points are indicated by crosshatched spheres. Oxygen octahedra are shown by dotted lines. Red spheres indicate oxygen and the blue spheres indicate the $B$ cations. Note that in this case, both electrical and mechanical boundary conditions are fulfilled. However, the atoms at the boundary are about to decide which domain their prefer, which causes disorder in atomic positions. This is crucial for domain switching and the magnitude of disorder depends on structural parameters. (b) One possibility to introduce long range order along the boundary is to allow finite width for the domain boundary by introducing a cubic layer. This also means that the electric polarization is zero at the boundary. Presumably this type of layer is formed in structures which do not significantly deviate from the cubic structure.

8 shows the experimental lattice parameters of PZT as a function of temperature. In the vicinity of the phase boundary the $c$ axis significantly shortens and the $a$ axis lengthens so that the $c / a$ axis ratio drops to one in the phase boundary area. Geometrical consideration shows that this makes it easier to match the pseudo-tetragonal (precisely, monoclinic $\mathrm{Cm}$ ) and rhombohedral crystals. This in turn suggests easier crystal boundary motion. 


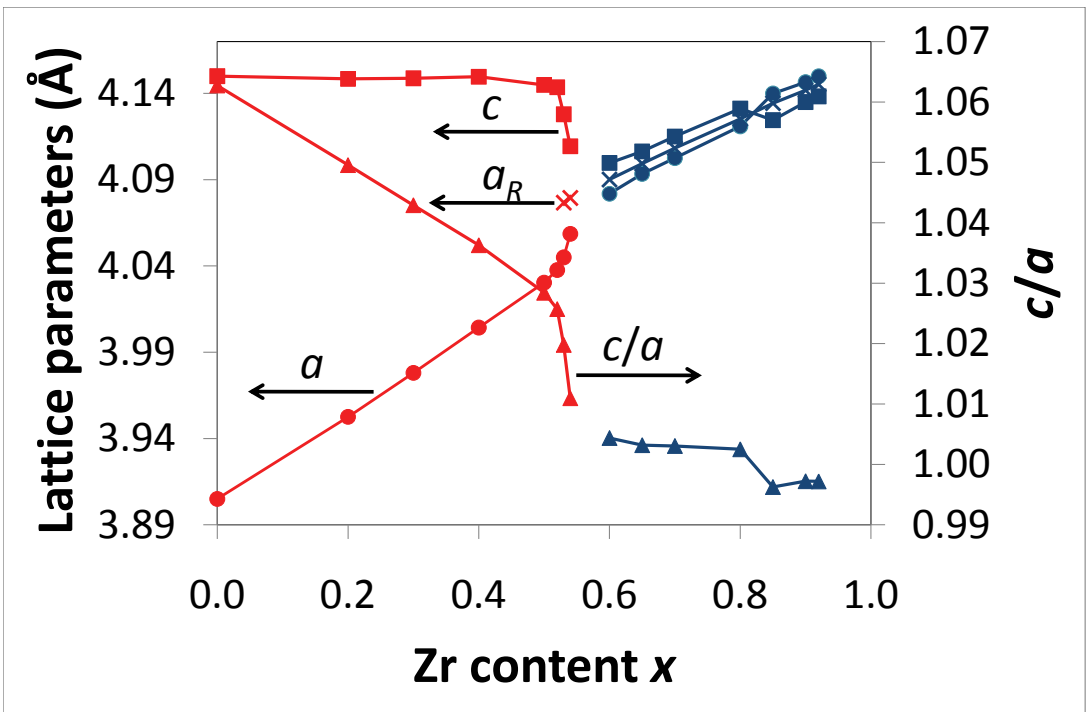

Fig. 8. Room-temperature tetragonal (space group $\mathrm{P} 4 \mathrm{~mm}$ ) and pseudo-tetragonal (space group $\mathrm{Cm}$ ) $a$ (filled spheres) and $c$ axis (filled squares) parameters and the $c / a$ axis ratio (filled triangles). Lattice parameter, $a_{R}$, for rhombohedral phase (space group $R 3 c$ ) is indicated by crosses. In the case of the monoclinic $\mathrm{Cm}$ phase (structure slightly deviates from the tetragonal structure) the average of the $a$ and $b$ axes were divided by $\sqrt{2}$, whereas $a_{R}=\frac{1}{3} \sqrt{3 a_{H}^{2}+\left(c_{H} / 2\right)^{2}}$, where $a_{H}$ and $c_{H}$ are the hexagonal lattice parameters. The data indicated by black colour are from. refs. (Frantti et al., 2000; 2002; 2003) and the data indicated by blue colour are from. ref. (Yokota, 2009).

\subsubsection{Domain wall width.}

We summarize the description given in ref. (Strukov \& Levanyuk, 1998) for a domain boundary (parallel to $y z$ plane) width estimation in a case that an infinitely large crystal is divided in two domains, one with $x<0$ and the other with $x>0$. To find out how the order parameter $\eta$ (in this case proportional to the spontaneous polarization) changes cross the boundary one includes a gradient term, proportional to $\left(\frac{\partial \eta}{\partial x}\right)^{2}$, to the density of the thermodynamic potential $\varphi(\eta)$. By expanding the thermodynamic potential up to forth order in order parameter and integrating over the entire crystal volume one gets the thermodynamic potential $\int_{v} \varphi(\eta) \mathrm{d} v=\int_{v}\left[\varphi_{0}+\frac{1}{2} A \eta^{2}+\frac{1}{4} B \eta^{4}+\frac{1}{2} C\left(\frac{\partial \eta}{\partial x}\right)^{2}\right] \mathrm{d} v$. A solution $\eta(x)$, which minimizes the thermodynamic potential, is obtained through variational computation with boundary conditions $\eta \rightarrow \eta_{0}$ when $x \rightarrow \infty$ and $\eta \rightarrow-\eta_{0}$ when $x \rightarrow-\infty$, where $\eta_{0}$ is a solution obtained for a homogeneous crystal. The solution is $\eta(x)= \pm \sqrt{-A / B} \tanh \left(\frac{x}{2 \sqrt{C /(-2 A)}}\right)$ (Strukov \& Levanyuk, 1998).

\section{Conclusions}

Piezoelectric contribution in lead-zirconate-titanate (PZT) ceramics was reviewed and classified to intrinsic and extrinsic contributions. Models of intrinsic contribution were 
addressed in light of recent experimental and theoretical studies. The very controversial polarization rotation model was addressed. Extrinsic contribution, consisted of grain boundary movement, domain wall movement, movement of the boundaries between crystal intergrowths and changes in phase fractions significantly contribute to the piezoelectric response of ceramics. Crystal symmetry analysis is not only useful for reducing the number of piezoelectric constants in single crystals, but finds applications in ferroelectric domain formation both in bulk ceramics and in thin films. Domain distribution depends on the sample size and shape, and the type of domain boundaries is affected by the sample preparation route. An example of the first case is Kittel's law, whereas changes in electrical conductivity between differently synthesized samples often result in different types of domain boundaries. Different contributions have characteristically different time-dependencies. Contemporary synchrotron facilities allow time-dependent studies down to $10 \mathrm{~ns}$, making time-dependent studies feasible.

\section{Acknowledgments}

This work was supported by the Academy of Finland (COMP Centre of Excellence Program 2006-2011) and Con-Boys Ltd. We are grateful to both of them.

\section{References}

Boysen, H. \& Altorfer, F. (1994). A Neutron Powder Investigation of the High-Temperature Structure and Phase Transition in $\mathrm{LiNbO}_{3}$. Acta Cryst. B, Vol. 50, 405-414.

Burns, G. \& Scott, B. A. (1970). Raman Studies of Underdamped Soft Modes in $\mathrm{PbTiO}_{3}$. Phys. Rev. Lett., Vol. 25, 167-170.

Camargo, E. R.; Leite, E. R. \& Longo, E. (2009). Synthesis and characterization of lead zirconate titanate powders obtained by the oxidant peroxo method. Journal of Alloys and Compounds, Vol. 469, 523-528.

Dawber, M; Rabe, K. M. \& Scott, J. F. (2005). Physics of thin-film ferroelectric oxides. Rev. Mod. Phys., Vol. 77, 1083-1130.

Dove, M. T. (2003). Structure and Dynamics: an Atomic View of Materials, Oxford University Press, ISBN 0-19-850678-3, New York.

Frantti, J.; Lantto, V.; Nishio, S. \& Kakihana, M. (1999). Effect of $A$ - and B-cation substitutions on the phase stability of $\mathrm{PbTiO}_{3}$ ceramics. Phys. Rev. B, Vol. 59, 12-15.

Frantti, J.; Lappalainen, J.; Lantto, V.; Nishio, S. \& Kakihana, M. (1999). Low-temperature Raman studies of $\mathrm{Pb}\left(\mathrm{Zr}_{x} \mathrm{Ti}_{1-x}\right) \mathrm{O}_{3}$ and $\mathrm{Pb}_{1-3 y / 2} \mathrm{Nd}_{y} \mathrm{TiO}_{3}$ ceramics. Jpn. J. Appl. Phys., Vol. 38, 5679-5682.

Frantti, J.; Lappalainen, J.; Eriksson, S.; Lantto, V.; Nishio, S.; Kakihana, M.; Ivanov, S. \& H. Rundlöf. (2000). Neutron diffraction studies of $\mathrm{Pb}\left(\mathrm{Zr}_{x} \mathrm{Ti}_{1-x}\right) \mathrm{O}_{3}$ ceramics. Jpn. J. Appl. Phys., Vol. 39, 5697-5703.

Frantti, J.; Ivanov, S.; Eriksson, S.; Rundlöf, H.; Lantto, V.; Lappalainen, J. \& Kakihana, M. (2002). Phase transitions of $\mathrm{Pb}\left(\mathrm{Zr}_{x} \mathrm{Ti}_{1-x}\right) \mathrm{O}_{3}$. Phys. Rev. B, Vol. 64, 064108.

Frantti, J.; Eriksson, S.; Hull, S.; Lantto, V.; Rundlöf, H. \& Kakihana, M. (2003). Composition variation and the monoclinic phase within $\mathrm{Pb}\left(\mathrm{Zr}_{x} \mathrm{Ti}_{1 x}\right) \mathrm{O}_{3}$ ceramics. J. Phys.: Condens. Matter, Vol. 15, No. 35, 6031-6041. 
Frantti, J.; Fujioka, Y. \& Nieminen, R. M. (2007). Pressure-Induced Phase Transitions in $\mathrm{PbTiO}_{3}$ : A Query for the Polarization Rotation Theory. J. Phys. Chem. B, Vol. 111, No. 17, 4287-4290.

Frantti, J.; Fujioka, Y. \& Nieminen, R. M. (2008). Evidence against the polarization rotation model of piezoelectric perovskites at the morphotropic phase boundary. J. Phys.: Condens. Matter, Vol. 20, No. 47, 472203-472207.

Frantti, J. (2008). Notes of the Recent Structural Studies on Lead Zirconate Titanate. J. Phys. Chem. B, Vol. 112, No. 21, 6521-6535.

Frantti, J.; Fujioka, Y.; Zhang, J.; Vogel, S. C.; Wang, Y.; Zhao, Y. \& Nieminen, R. M. (2009). The Factors Behind the Morphotropic Phase Boundary in Piezoelectric Perovskites. J. Phys. Chem. B, Vol. 113, No. 23, 7967-7972.

Fu, H. \& Cohen, R. E. (2000). Polarization rotation mechanism for ultrahigh electromechanical response in single-crystal piezoelectrics. Nature Letters, Vol. 403, 281-283.

Glazer, A. M.; Thomas, P. A.; Baba-Kiski, K. Z.; Pang, G. K. H. \& Tai, C. W. (2004). Influence of short-range and long-range order on the evolution of the morphotropic phase boundary in $\mathrm{Pb}\left(\mathrm{Zr}_{1-x} \mathrm{Ti}_{x}\right) \mathrm{O}_{3}$. (2004). Phys. Rev. B, Vol. 70, 184123.

Grigoriev, A.; Sichel, R.; Lee, H. N., Landahl, E. C.; Adams, B.; Dufresne, E. M. \& Evans, P. G. (2008). Nonlinear Piezoelectricity in Epitaxial Ferroelectrics at High Electric Fields. Phys. Rev. Lett., Vol. 100, 027604.

Grigoriev, A.; Sichel, R. J.; Jo, J. Y.; Choudhury, S.; Chen, L-Q.; Lee, H. N.; Landahl, E. C.; Adams, B. W.; Dufresne, E. M. \& Evans, P. G. (2009). Stability of the unswitched polarization state of ultrathin epitaxial $\mathrm{Pb}(\mathrm{Zr}, \mathrm{Ti}) \mathrm{O}_{3}$ in large fields. Phys. Rev. B, Vol. 80, 014110 .

Hahn, Th. \& Klapper, H. (2003). Twinning of Crystals, In: International Tables for Crystallography D: Physical Properties of Crystals, A. Authier, (Ed.), 393-448, Kluwer Academic Publishers, ISBN 1-4020-0714-0, Dordrecht.

Hahn, Th. \& Klapper, H. (2005). International Tables for Crystallography A: Space-group Symmetry, Th. Hahn, (Ed.), Kluwer Academic Publishers, ISBN 0-7923-6590-9, Dordrecht.

Hinterstein, M.; Hoelzel, M.; Kungl, H.; Hoffmann, M. J.; Ehrenberg, H. \& Fuess, H. (2011). In situ neutron diffraction study of electric field induced structural transitions in lanthanum doped lead zirconate titanate. Zeitschrift Fur Kristallographie, Vol. 226, 155-162.

Hoffmann, M. J. \& Kungl, H. (2004). High strain lead-based perovskite ferroelectrics. Current Opinion in Solid State and Materials Science, Vol. 8, 51-57.

Hsu, R.; Maslen, E. N.; Du Boulay, D. \& Ishizawa, N. (1997). Synchrotron X-ray Studies of $\mathrm{LiNbO}_{3}$ and $\mathrm{LiTaO}_{3}$. Acta Cryst. B, Vol. 53, 420-428.

Jaffe, B.; Cook, W. R. \& Jaffe, H. (1971). Piezoelectric Ceramics, Academic Press, ISBN 0123795508, New York.

Janolin, P.-E. (2009). Strain on ferroelectric thin films. J. Mater. Sci., Vol., 44, 5025-5048.

Kano, J.; Tsukada, S.; Zhang, F.; Karaki, T.; Adachi, M. \& Kojima, S. (2007). Characterization of Dielectric Property of Nanosized $\left(\mathrm{Pb}_{0.7} \mathrm{Sr}_{0.3}\right) \mathrm{TiO}_{3}$ Powders Studied by Raman Scattering. Jpn. J. Appl. Phys., Vol. 46, No. 10B, 7148-7150.

Kittel, C. (1946). Theory of the Structure of Ferromagnetic Domains in Films and Small Particles. Phys. Rev., Vol. 70, 965-971. 
Klapper, H \& Hahn, Th. (2005). Point-group Symmetry and Physical Properties of Crystals, In: International Tables for Crystallography A: Space-group Symmetry, Th. Hahn, (Ed.), 804-808, Kluwer Academic Publishers, ISBN 0-7923-6590-9, Dordrecht.

Koch, E. (2004). Twinning, In: International Tables for Crystallography C: Mathematical, Physical and Chemical Tables, E. Prince, (Ed.), 10-14, Kluwer Academic Publishers, ISBN 1-4020-1900-9, Dordrecht.

Kolasinski, K. W. (2008). Surface Science: Foundations of Catalysis and Nanoscience, John Wiley \& Sons Ltd, ISBN 978-0-470-03308-1, West Sussex, England.

Kozielski, L; Buixaderas, E.; Clemens, F. \& Bujakiewicz-Korónska. (2010). PZT Microfibre defect structure studied by Raman spectroscopy. J. Phys. D: Appl. Phys., Vol. 43, 415401.

Jones, J. L.; Hoffman, M.; Daniels, J. E. \& Studer, A. J. (2006). Direct measurement of the domain switching contribution to the dynamic piezoelectric response in ferroelectric ceramics. Appl. Phys. Lett., Vol. 89, No. 9, 092901.

Landauer, R. (1957). Electrostatic Considerations in $\mathrm{BaTiO}_{3}$ Domain Formation During Polarization Reversal. J. Appl. Phys., Vol. 28, 227-234.

Li, J. Y.; Rogan, R. C.; Üstündag, E. \& Bhattacharya, K. (2005). Domain switching in polycrystalline ferroelectric ceramics. Nature Materials, Vol. 4, 776-781.

Lines, M. E. \& Glass, A. M. (2001). Principles and Applications of Ferroelectrics and Related Materials, Oxford University Press in Oxford Classic Series, ISBN 0-19-850778-X, Oxford.

Liu, Y. Y.; Zhu, Z. X.; Li, J.-F. \& Li, J. Y. (2010). Misfit strain modulated phase structures of epitaxial $\mathrm{Pb}\left(\mathrm{Zr}_{1-x} \mathrm{Ti}_{x}\right) \mathrm{O}_{3}$ thin films: The effect of substrate and film thickness. Mechanics of Materials, Vol. 42, 816-826.

Lüth, H. (2001). Solid Surfaces, Interfaces and Thin Films, Springer-Verlag, ISBN 3-540-58576-1, Berlin, Heidelberg.

Mitsui, T. \& Furuichi, J. (1953). Domain Structure of Rochelle Salt and $\mathrm{KH}_{2} \mathrm{PO}_{4}$. Phys. Rev., Vol. 90, 193-202.

Newnham, R. E. (2005). Properties of Materials: Anisotropy, Symmetry, Structure, Oxford University Press, ISBN 0-19-852076-X, New York.

Nishida, K.; Osada, M.; Wada, S.; Okamoto, S.; Ueno, R.; Funakubo, H. \& Katoda, T. (2005). Raman Spectroscopic Characterization of Tetragonal $\mathrm{PbZr}_{x} \mathrm{Ti}_{1-x} \mathrm{O}_{3}$ Thin Films: A Rapid Evaluation Method for c-Domain Volume. Jpn. J. Appl. Phys., Vol. 25, No. 25, L827-L829.

Noheda, B.; Cox, D. E.; Shirane, G.; Gonzalo, J. A.; Cross, L. E. \& Park, S.-E. (1999). A monoclinic ferroelectric phase in the $\mathrm{Pb}\left(\mathrm{Zr}_{1-x} \mathrm{Ti}_{x}\right) \mathrm{O}_{3}$ solid solution. Appl. Phys. Lett., Vol. 74, 2059-2061.

Noheda, B.; Gonzalo, J. A.; Cross, L. E.; Guo, R.; Park, S.-E.; Cox, D. E. \& Shirane, G. (2000). Tetragonal-to-monoclinic phase transition in a ferroelectric perovskite: The structure of $\mathrm{PbZr}_{0.52} \mathrm{Ti}_{0.48} \mathrm{O}_{3}$. Phys. Rev. B, Vol. 61, 8687-8695.

Nye, J. F. (1995). Physical Properties of Crystals, Oxford University Press, ISBN 0-19-851165-5, New York.

Ohno, T.; Matsuda, T.; Ishikawa, K. \& Suzuki, H. (2006). Thickness Dependence of Residual Stress in Alkoxide-Derived $\mathrm{Pb}\left(\mathrm{Zr}_{0.3} \mathrm{Ti}_{0.7}\right) \mathrm{O}_{3}$ Thin Film by Chemical Solution Deposition. Jpn. J. Appl. Phys., Vol. 45, No. 9B, 7265-7269. 
Olsen, R. B. \& Evans, D. (1983). Pyroelectric energy conversion: Hysteresis loss and temperature sensitivity of a ferroelectric materials. J. Appl. Phys., Vol. 54, 5941-5944.

Pane, I.; Fleck, N. A.; Huber, J. E. \& Chu, D. P. (2008). Effect of geometry upon the performance of a thin film ferroelectric capacitor. International Journal of Solids and Structures, Vol $45,2024-2041$.

Pertsev, N. A. \& Zembilgotov, A. G. (1995). Energetics and geometry of $90^{\circ}$ domain structures in epitaxial ferroelectric and ferroelastic films. J. Appl. Phys., Vol. 78, 6170-6180.

Phelan, D.; Long, X.; Xie, Y.; Ye, Z.-G.; Glazer, A. M.; Yokota, H.; Thomas, P. A. \& Gehring, P. M. (2010). Single Crystal Study of Competing Rhombohedral and Monoclinic Order in Lead Zirconate Titanate. Phys. Rev. Lett., Vol. 105, 207601.

Phillips, R. B. (2001). Crystals, Defects and Microstructures: Modeling Across Scales, Cambridge University Press, ISBN 0-521-79357-2, Cambridge.

Pramanick, A.; Damjanovic, D.; Nino, J. C. \& Jones, J. (2009). Subcoercive Cyclic Electrical Loading of Lead Zirconate Titanate Ceramics I: Nonlinearities and Losses in the Converse Piezoelectric Effect. J. Am. Ceram. Soc., Vol. 92, No. 10, 2291-2299.

Pramanick, A.; Daniels, J. E. \& Jones, J. (2009). Subcoercive Cyclic Electrical Loading of Lead Zirconate Titanate Ceramics II: Time-resolved X-ray Diffraction. J. Am. Ceram. Soc., Vol. 92, No. 10, 2300-2310.

Pruvost, S.; Hajjaji, A.; Lebrun, L.; Guyomar, D. \& Boughaleb, Y. (2010). Domain switching and Energy Harvesting Capabilities in Ferroelectric Materials. J. Phys. Chem. C, Vol. 114, 20629-20635.

Ricote, J.; Corker, D. L.; Whatmore, R. W.; Impey, S. A.; Glazer, A. M.; Dec, J. \& Roleder, K. (1998). A TEM and neutron diffraction study of the local structure in the rhombohedral phase of lead zirconate titanate. J. Phys.: Condens. Matter, Vol. 10, 1767-1786.

Rogan, R. C.; Üstündag, E.; Clausen, B. \& Daymond M. R. (2003). Texture and strain analysis of the ferroelastic behavior of $\mathrm{Pb}(\mathrm{Zr}, \mathrm{Ti}) \mathrm{O}_{3}$ by in situ neutron diffraction. J. Appl. Phys., Vol. 93, No. 7, 4104-4111.

Roitburd, A. L. (1976). Equilibrium Structure of Epitaxial Layers. Phys. Status Solidi A, Vol. 37, 329-339.

Schilling, A.; Adams, T. B.; Bowman, R. M.; Gregg, J. M.; Catalan, G. \& Scott, J. F. (2006). Scaling of domain periodicity with thickness measured in $\mathrm{BaTiO}_{3}$ single crystal lamellae and comparison with other ferroics. Phys. Rev. B, Vol. 74, 024115.

Sergienko, I. A.; Gufan, Y. M. \& Urazhdin, S. (2002). Phys. Rev. B, Vol. 65, 144104.

Souza Filho, A. G.; Lima, K. C. V.; Ayala, A. P.; Guedes, I.; Freire, P. T. C.; Melo, F. E. A., Mendes Filho, J.; Araújo, E. B. \& Eiras, J. A. (2002). Raman scattering study of the $\mathrm{PbZr}_{1-x} \mathrm{Ti}_{x} \mathrm{O}_{3}$ system: Rhombohedral-monoclinic-tetragonal phase transitions. Phys. Rev. B, Vol. 66, 132107.

Streiffer, S. K.; Eastman,J. A.; Fong, D. D.; Thompson, C.; Munkholm, A.; Ramana Murty, M. V.; Auciello, O.; Bai, G. R. and Stephenson, G. B. (2002). Observation of Nanoscale $180^{\circ}$ Stripe Domains in Ferroelectric $\mathrm{PbTiO}_{3}$ Thin Films. Phys. Rev. Lett., Vol. 89, 067601.

Strukov, B. A. \& Levanyuk, A. P. (1998). Ferroelectric Phenomena in Crystals: Physical Foundations, Springer-Verlag, ISBN 3-540-63132-1, Berlin. 
Thomas, N. W. \& Beitollahi, A. (1994). Inter-Relationship of Octahedral Geometry, Polyhedral Volume Ratio and Ferroelectric Properties in Rhombohedral Perovskites. Acta Cryst. B, Vol. 50, 549-560.

Topolov, V. Y. \& Turik, A. V. (2001). A new monoclinic phase and features of stress relief in $\mathrm{PbZr}_{1-x} \mathrm{Ti}_{x} \mathrm{O}_{3}$ solid solutions. J. Phys. Condens. Matter, Vol. 13, L771-L775.

Yakunin, S. I.; Shakmanov, V. V.; Spivak, G. V. \& Vasiléva, N. V. (1972). Microstructure of domains and domain boundaries of $\mathrm{BaTiO}_{3}$ single crystalline films. Fiz. Tverd. Tela, Vol. 14, 373-377, Sov. Phys. Solid State (English Transl.), Vol. 14, 310.

H. Yokota, N. Zhang, A. E. Taylor, P. Thomas, and A. M. Glazer (2009). Crystal structure of the rhombohedral phase of $\mathrm{PbZr}_{1-x} \mathrm{Ti}_{x} \mathrm{O}_{3}$ ceramics at room temperature Phys. Rev. B, Vol. 80, 104109.

Wu, X. \& Vanderbilt, D. (2006). Theory of hypothetical ferroelectric superlattices incorporating head-to-head and tail-to-tail $180^{\circ}$ domain walls. Phys. Rev. B, Vol. 73, 020103.

Xu, G.; Wen, J.; Stock, C. \& Gehring, P. M. (2008). Phase instability induced by polar nanoregions in a relaxor ferroelectric system. Nature Materials, Vol. 7, 562-566. 


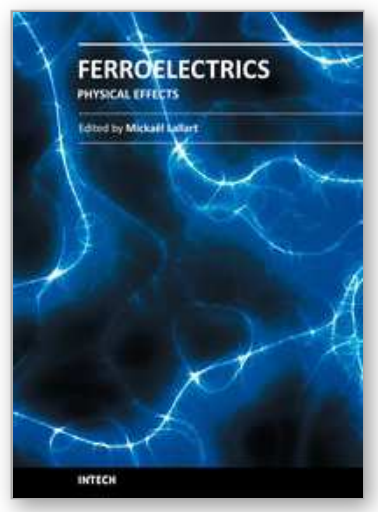

\author{
Ferroelectrics - Physical Effects \\ Edited by Dr. MickaÃ «l Lallart
}

ISBN 978-953-307-453-5

Hard cover, 654 pages

Publisher InTech

Published online 23, August, 2011

Published in print edition August, 2011

Ferroelectric materials have been and still are widely used in many applications, that have moved from sonar towards breakthrough technologies such as memories or optical devices. This book is a part of a four volume collection (covering material aspects, physical effects, characterization and modeling, and applications) and focuses on the underlying mechanisms of ferroelectric materials, including general ferroelectric effect, piezoelectricity, optical properties, and multiferroic and magnetoelectric devices. The aim of this book is to provide an up-to-date review of recent scientific findings and recent advances in the field of ferroelectric systems, allowing a deep understanding of the physical aspect of ferroelectricity.

\title{
How to reference
}

In order to correctly reference this scholarly work, feel free to copy and paste the following:

Johannes Frantti and Yukari Fujioka (2011). Piezoelectricity in Lead-Zirconate-Titanate Ceramics - Extrinsic and Intrinsic Contributions, Ferroelectrics - Physical Effects, Dr. MickaÃ«I Lallart (Ed.), ISBN: 978-953-307453-5, InTech, Available from: http://www.intechopen.com/books/ferroelectrics-physical-effects/piezoelectricityin-lead-zirconate-titanate-ceramics-extrinsic-and-intrinsic-contributions

\section{INTECH}

open science | open minds

\section{InTech Europe}

University Campus STeP Ri

Slavka Krautzeka 83/A

51000 Rijeka, Croatia

Phone: +385 (51) 770447

Fax: +385 (51) 686166

www.intechopen.com

\section{InTech China}

Unit 405, Office Block, Hotel Equatorial Shanghai

No.65, Yan An Road (West), Shanghai, 200040, China

中国上海市延安西路65号上海国际贵都大饭店办公楼405单元

Phone: +86-21-62489820

Fax: +86-21-62489821 
(C) 2011 The Author(s). Licensee IntechOpen. This chapter is distributed under the terms of the Creative Commons Attribution-NonCommercialShareAlike-3.0 License, which permits use, distribution and reproduction for non-commercial purposes, provided the original is properly cited and derivative works building on this content are distributed under the same license. 